\title{
Magnetic Resonance Imaging Procedure for Ventilated Intensive Care Patient in the International Islamic University Malaysia Medical Centre: Issues and Challenges
}

\author{
Nazhan Afeef Mohd. Ariff @ Ghazali ${ }^{1}$, Iqbalmunauwir Ab Rashid ${ }^{1}$ \\ ${ }^{1}$ Department of Anaesthesiology and Intensive Care, Kulliyyah of Medicine, \\ International Islamic University Malaysia
}

Presenter: Nazhan Afeef Mohd. Ariff @ Ghazali

Managing a ventilated patient in the Intensive Care Unit (ICU) for Magnetic Resonance Imaging (MRI) procedure requires precise planning. Vigilant anesthetist with adequate MRI compatible monitoring of a critically ill patient and in preparation of any crisis event during transport to MRI suite, during MRI and return journey to ICU is essential. Problems in transporting ICU patient are usually equipment related, deterioration in gas exchange and increased rate of ventilator associated pneumonia. Preparation includes clinical reassessment for swift and systematic transport, airway management, optimum sedation and record keeping close to the patient. This is a case report of a 64 year-old gentleman who presented to International Islamic University Malaysia Medical Centre with right sided headache for one week associated with high intracranial pressure symptoms. His initial Glasgow Coma Scale (GCS) was 15/15 and Computed Tomography (CT) scan of his brain showed bilateral acute-on-chronic subdural haemorrhage, uncal herniation and minimal midline shift. His GCS dropped to 6/15 $\left(E_{1} V_{1} M_{4}\right)$ in the ward and proceeded with right craniectomy, evacuation of clot and duraplasty. Post operatively, his GCS improved to $15 / 15$ in ICU and was extubated. On Day 2 post extubation, his GCS dropped to 6/15 $\left(E_{1} V_{1} M_{4}\right)$ and intubation was performed. Repeat CT Brain in ICU showed no evidence of new bleeding or mass effect. MRI was performed and it showed acute infarctions at right pons and right occipital region. Tracheostomy was done and the patient was sent to the general ward on fixed performance mask. 